ज्ञ FRANÇAISE

$\ \mathrm{DE}$

밈EAGOGIE

\section{Revue française de pédagogie}

Recherches en éducation

155 | avril-juin 2006

La motivation scolaire : approches récentes et perspectives pratiques

\title{
Les buts sociaux de l'élève : leurs causes et leurs conséquences à l'école
}

The Social Goals of Students: Causes and Consequences at School

Los objetivos sociales del alumno: sus causas y sus consecuencias en la escuela

Die Soziale Ziele der Schulern: Ursachen und Konsequenzen in der Schule

Laurence Filisetti, Kathryn Wentzel et Éric Dépret

\section{(2) OpenEdition \\ Journals}

\section{Édition électronique}

URL : http://journals.openedition.org/rfp/220

DOI : $10.4000 /$ rfp. 220

ISSN : 2105-2913

Éditeur

ENS Éditions

\section{Édition imprimée}

Date de publication : 1 juin 2006

Pagination : 45-56

ISBN : 978-2-7342-1047-4

ISSN : 0556-7807

Référence électronique

Laurence Filisetti, Kathryn Wentzel et Éric Dépret, «Les buts sociaux de l'élève : leurs causes et leurs conséquences à l'école », Revue française de pédagogie [En ligne], 155 | avril-juin 2006, mis en ligne le 21 septembre 2010, consulté le 30 avril 2019. URL : http://journals.openedition.org/rfp/220 ; DOI : $10.4000 /$ rfp. 220 


\section{Les buts sociaux de l'élève : leurs causes et leurs conséquences à l'école}

\section{Laurence Filisetti, Kathryn Wentzel, Éric Dépret}

Les recherches en psychologie sociale appliquée à l'éducation se sont largement intéressées au domaine des buts et à leurs influences sur les performances. Loin de dénigrer leur importance dans les apprentissages, ces recherches se réfèrent majoritairement à une dimension cognitive des buts - s'exprimant par le désir d'apprendre ou d'être meilleur que les autres - plutôt qu'à une dimension sociale - s'exprimant, elle, par le désir de se faire accepter des autres (Wentzel, 2002a). Cet article se propose, en conséquence, de montrer l'importance des buts sociaux dans les apprentissages scolaires. Après une définition des buts sociaux et de la manière dont ils sont opérationnalisés dans les recherches, nous développerons le rôle que peut jouer la poursuite de buts sociaux de l'élève à l'école. Les facteurs qui peuvent déterminer ces buts seront ensuite abordés à travers une synthèse de nos recherches. Enfin des implications pour la pratique seront développées.

Descripteurs (TEE) : comportement de l'élève, conditions d'apprentissage, perception de soi, performance, réussite scolaire.

es recherches en psychologie sociale appliquée à l'éducation se sont largement intéressées à la notion de buts ainsi qu'à leurs influences sur les performances des élèves. Si une grande majorité des études se focalise sur les buts qualifiés d'académiques (réussir en mathématiques par exemple) peu, en revanche, examinent l'impact des buts sociaux à l'école. L'objectif de cet article consiste, alors, à présenter en quoi la poursuite de tels buts peut être importante à l'école et quels sont les facteurs qui expliquent qu'un enfant les poursuive. À l'heure où "le savoir-vivre ensemble" est l'une des priorités de l'école, une meilleure connaissance de la motivation de l'enfant dans ses efforts avec les autres peut s'avérer, comme nous allons le voir, fondamentale.

\section{DÉFINITION ET OPÉRATIONNALISATION DES BUTS SOCIAUX}

De manière générale et bien que les définitions varient en fonction des perspectives théoriques, les buts peuvent se définir comme des représentations cognitives d'un individu concernant les résultats qu'il 
aimerait atteindre ou accomplir suite à certains efforts fournis (Ford, 1996; Pintrinch \& Schunk, 1996 ; Wentzel, 1991b \& 1994). Ces buts que la personne établit pour elle-même peuvent être de puissants éléments jouant sur son interprétation et sa réaction aux événements, ainsi que sur les comportements qu'elle va mettre en œuvre ou éviter (Austin \& Vancouver, 1996 ; Bandura, 1986 ; Dweck, 1991 ; Dweck \& Leggett, 1998 ; Ford, 1996). Plus spécifiquement, dans le domaine éducatif, les buts ont été majoritairement abordés en termes " académiques", c'est-à-dire comme des orientations qui guident les comportements que l'élève adopte face à une tâche scolaire (par ex. : Dweck, 2002 ; Nicholls, 1984). Ces orientations prennent deux formes. L'élève peut être motivé pour être meilleur que les autres ou plus généralement pour obtenir des jugements favorables au regard de ses performances. II est orienté, alors, vers des buts de performance. II peut également être motivé pour développer ses connaissances et ses compétences dans un domaine : il est, cette fois-ci, orienté vers des buts dits d'apprentissage ou de maîtrise (par ex. : Dweck, 2002 ; Dweck \& Leggett, 1998 ; Harachiewicz \& Elliot, 1993 ; Nicholls, 1984). Ce n'est plus rarement que les études conduites dans le milieu scolaire s'intéressent aux « orientations sociales " de l'élève. Parmi les travaux traitant de ce thème, ceux de Wentzel constituent désormais une référence. Les buts sociaux y sont définis, de manière générale, comme des résultats que l'élève cherche à atteindre dans ses interactions avec les autres (par ex.: Wentzel, 2002a). Plus spécifiquement, deux types de buts sociaux sont plus particulièrement suivis à l'école. D'une part, il y a les buts «prosociaux » qui permettent la mise en place de comportements tels que l'aide, la coopération ou le partage. D'autre part, il y a les buts dits de «responsabilité sociale " qui sous-tendent l'adoption de comportements tels que respecter des règles, tenir ses promesses ou ses engagements (par ex.: Wentzel, 2003).

De ce fait, pour évaluer les buts sociaux de l'élève, sont mesurés les efforts qu'il dit fournir dans le domaine prosocial ainsi que dans le domaine de la responsabilité sociale. C'est, à ce propos, l'échelle mise en place par Wentzel, en 1993, qui semble l'outil le plus largement utilisé. Cette échelle est constituée de treize items comme «Est-ce qu'il t'arrive souvent de partager ton matériel avec tes camarades ? " (exemple d'item prosocial) ou "Est-ce qu'il t'arrive souvent de continuer de travailler alors que tes camarades se dissipent? " (exemple d'item de responsabilité sociale). Les élèves y répondent en entourant un chiffre allant de 1 à 6 où 1correspond à «rarement" et où 6 correspond à "presque toujours ».

\section{LES CONSÉQUENCES DES BUTS SOCIAUX À L'ÉCOLE}

En quoi est-il pertinent de s'intéresser aux buts sociaux que l'élève poursuit à l'école? De manière générale, les buts qu'une personne poursuit affectent ses performances (Ford, 1984). Ils fournissent de l'impulsion à ses actions et les dirigent (Pintrich \& Schunk, 1996). Ainsi, pour atteindre l'objectif qu'elle s'est fixée, la personne va orienter son attention, son énergie, son intérêt vers différentes tâches plutôt que d'autres. Elle va privilégier certaines stratégies et faire preuve de persévérance pour atteindre le but qu'elle a en tête (Ford, 1984). En conséquence, tous ces éléments qui sont l'intérêt, la persévérance, l'attention et le choix des stratégies (ou plus globalement son comportement face à une tâche) vont concourir à influencer sa performance.

Des buts plus spécifiques comme le sont les buts sociaux, n'échappent pas à la règle. De nombreuses études révèlent en effet un lien entre la poursuite des buts sociaux des élèves et leurs comportements ainsi que leurs performances en classe.

Plus concrètement, des travaux ont montré que les enfants qui réussissent le mieux en classe, c'est-àdire les élèves qui obtiennent de meilleures notes, sont aussi ceux qui poursuivent des buts sociaux (Wentzel, 1989 \& 1993). Ils arrivent en classe à l'heure, essaient de participer, de faire des efforts pour réaliser le travail demandé et tentent enfin de ne pas déranger la classe (Finn \& Rock, 1997). Mieux encore, ceux qui poursuivent le plus de buts (buts académiques et sociaux) sont les meilleurs élèves ( $87 \%$ d'entre eux) tandis que leurs camarades en difficulté scolaire (67\% d'entre eux) ne déclarent poursuivre qu'un seul et unique but, celui de s'amuser (Wentzel, 1991b). En d'autres termes, ne pas faire d'efforts pour gagner l'approbation des autres ou pour être socialement responsable, c'est-à-dire ne pas essayer d'adopter des comportements valorisés par les enseignants et les pairs (Wentzel, 2003) caractérisent les moins bons élèves (Wentzel, 1991b). D'autres études démontrent que plus qu'une simple caractéristique des bons élèves, la poursuite 
de plusieurs buts sociaux peut prédire les performances scolaires. Ainsi, des études conduites auprès de collégiens révèlent que les buts sociaux poursuivis par un élève prédisent les efforts qu'il consent à fournir dans une activité de classe (en termes de temps passé sur ses leçons et d'attention prêtée en classe (Wentzel, 1996), efforts qui peuvent influencer ses performances. Des études longitudinales ajoutent, enfin, que les buts sociaux que l'enfant poursuit en classe de sixième permettent de prédire les performances scolaires qu'il aura une année plus tard (Wentzel, 1998).

Comment ce lien entre une dimension sociale (les buts) et une dimension académique (la performance) peut-il se comprendre? Une première explication, d'ordre cognitif, peut être donnée. Certains auteurs, tel que Ford (1982), insistent sur le fait que ces deux dimensions ne peuvent pas être considérées indépendamment l'une de l'autre. Un élève qui fait des efforts dans ses relations avec les autres et qui parvient alors à adopter des comportements appropriés avec eux, est un élève qui possède des ressources cognitives importantes. En effet, résoudre des problèmes avec ses camarades, partager avec eux, comprendre leurs émotions, les écouter ou anticiper les conséquences de leurs actions demandent certaines capacités cognitives. Non seulement ces capacités vont lui être utiles dans ses relations avec les autres mais elles l'aideront également à réaliser certains exercices scolaires. Par exemple, l'énergie mobilisée pour écouter son ami peut être exploitée également pour écouter une leçon. De fait, nous pouvons mieux comprendre pourquoi un élève qui poursuit des buts sociaux est aussi un bon élève : il possède certaines capacités cognitives qu'il peut transférer du domaine social vers le domaine scolaire.

Une seconde explication, d'ordre social quant à elle, peut être donnée. Pour certains auteurs, le rapport qu'entretiennent les buts sociaux de l'élève et ses performances scolaires peut être compris à travers l'étude des relations élèves-enseignant. Plus exactement, il a été montré qu'il existe un lien entre certains buts sociaux poursuivis par l'élève et combien il est apprécié par le professeur. Concrètement, un élève qui fournit des efforts notamment pour réaliser le travail demandé va adopter, en classe, certains comportements tels que le partage, l'aide ou la coopération. Ces comportements suscitent l'approbation du professeur puisque celui-ci déclare vouloir encore travailler avec cet élève l'année suivante (Wentzel, 1994). Autrement dit, les élèves qui sont davantage appréciés des enseignants sont avant tout ceux qui font des efforts pour respecter certaines règles (des règles de travail par exemple) et qui mettent alors en œuvre certains comportements (Wentzel, 1991b). Plusieurs recherches abondent dans ce sens et confirment que les enseignants ressentent plus d'attachement et moins d'indifférence envers les élèves que l'on pourrait qualifiés de conformes (Helton \& Oakland, 1977). Ils les jugent comme plus sympathiques et meilleurs élèves (par ex.: Dépret \& Filisetti, 2001 pour une étude sur la politesse). Les répercussions que ces préférences et ces impressions peuvent avoir sur les performances peuvent être aisément envisagées. Le ressenti de l'enseignant quant aux élèves, les facultés que ses jugements ont de s'auto-réaliser (par ex. : Bressoux \& Pansu, 2003) peuvent affecter son comportement pédagogique. La clarté de ses consignes, la dureté des sanctions ou la quantité des récompenses, le degré d'investissement ou encore l'énergie déployée pour aider les enfants peuvent être touchés (par ex. : Rohrkemper \& Brophy 1983). En conséquence, par les buts sociaux que l'élève poursuit, par le lien indirect qu'ils entretiennent avec le ressenti du professeur, les performances et les apprentissages peuvent être touchés.

Mais ce n'est pas tout, certains travaux insistent aussi sur le fait que l'impact des buts sociaux sur les pertormances peut s'expliquer par la relation que l'élève entretient avec ses pairs. Tout comme dans la relation avec l'enseignant, la poursuite de certains buts sociaux par l'élève est liée à son acceptation par les pairs. En faisant des efforts pour partager avec les autres ce qu'il a appris (buts prosociaux), l'élève adopte des comportements coopératifs et aidants qui le font apprécier des autres. Ceux-ci déclarent en effet vouloir à nouveau échanger avec lui (Wentzel, 1994). Et être apprécié, ou au contraire rejeté par ses pairs n'est pas sans importance à l'école. L'impopularité peut être lourde de conséquences pour un enfant. Non seulement percu comme déviant par l'adulte, l'impopulaire est vu par ses camarades comme quelqu'un qui dérange la bonne marche du groupe et comme un mauvais élève (Coie, Dodge \& Coppotelli, 1983). Il s'estime plus déprimé que les autres (Vosk et al., 1982). Son intérêt pour les activités de classe est faible (Wentzel, 1998 ; Wentzel \& Asher, 1995) et peut l'amener plus vite que ses camarades à délaisser les bancs de l'école (Parker \& Asher, 1987). Nous pouvons imaginer, en effet, combien il doit être peu enthousiasmant de se rendre en classe tous les matins sachant 
qu'il va falloir affronter les quolibets des uns et jouer des poings avec les autres. Les notions enseignées risquent de ne pas être correctement assimilées par ces enfants bien trop préoccupés par leurs soucis relationnels. L'enfant populaire, lui, est dans une toute autre situation. Avoir des amis, appartenir à un groupe lui confère de bien nombreux avantages. Aimé de tous, il peut, en effet, sans aucune réticence, demander des éclaircissements à l'un, de l'aide à l'autre, (Wentzel, 1991a; Wentzel \& Cadwell, 1997) ou encore recevoir de nombreux renforcements positifs (Gottman, Gonso \& Rasmussen, 1975). Autant d'éléments ou d'informations qui peuvent favoriser ses apprentissages en classe.

Ainsi comme nous venons de le voir, la poursuite ou non de buts sociaux n'est pas sans importance à l'école. Elle influence les comportements de l'enfant, ce qui a pour conséquences d'agir sur la façon dont il sera aimé des autres (pairs et enseignants) et dont il sera aidé. Son intérêt pour les activités scolaires et ses performances s'en trouveront alors indirectement affectés.

La question que les chercheurs se posent désormais est de savoir ce qui explique qu'un enfant s'engage activement dans la poursuite de buts sociaux? Mieux connaître ce qui détermine ces buts c'est aussi mieux envisager comment aider l'élève à les poursuivre pour ainsi mieux le faire progresser dans ses comportements avec les autres et dans ses apprentissages.

\section{LES DÉTERMINANTS DES BUTS SOCIAUX DE L'ÉLĖVE}

\section{Un point sur la littérature}

Alors que le l'impact des buts sociaux des élèves a été largement examiné, l'étude, elle, de leurs déterminants a été négligée. Des recherches que nous avons récemment menées s'y sont donc employées amenant ainsi les premiers éléments de réponses dans ce domaine (Wentzel, Filisetti, \& Looney, à paraître). Dans notre travail visant à mettre en évidence les facteurs à l'origine des buts sociaux, deux approches théoriques ont suscité plus particulièrement notre intérêt. Toutes deux examinent l'influence que peut avoir les perceptions de l'individu sur ses efforts. II s'agit d'une part du modèle de Connell et Wellborn (1991) et d'autre part, de la théorie de l'autodétermination (par ex. : Deci \& Ryan, 1991).
Dans le modèle motivationnel qu'ils présentent en 1991, Connell et Wellborn stipulent que l'investissement que l'individu déploie pour réaliser une tâche peut s'expliquer par les perceptions qu'il a de luimême et des autres. De manière générale, selon les auteurs, toute personne évalue ses actions au regard de trois besoins psychologiques fondamentaux: le besoin de compétence (signifiant se sentir capable de), le besoin d'autonomie (signifiant se sentir à l'initiative de ses actes) et le besoin de lien (signifiant se sentir soutenu, aidé par les autres). Selon que l'individu se perçoit satisfait ou non quant à ces dimensions, les efforts mis en œuvre sont différents. Dans le domaine plus spécifique qu'est l'école, l'élève peut évaluer la satisfaction de ces besoins fondamentaux de la façon suivante. Pour déterminer s'il est compétent, il peut se poser la question : « Est-ce que je sais ce qu'il faut faire pour réussir à l'école ? " et "Est-ce que j'en suis capable ?". Ensuite, pour évaluer son besoin d'autonomie, l'élève peut se demander à quel point il est à l'initiative de ses apprentissages (par ex.: "Est-ce que je travaille à l'école parce que c'est important » ou « pour éviter d'être puni(e) ? »). Enfin pour évaluer à quel point il est lié aux autres, l'élève peut se demander : "Estce que je me sens bien dans mes relations avec les autres?". Pour Connell et Wellborn (1991), les réponses à ces questions ont différentes implications sur la motivation de l'élève. II peut faire preuve de désinvestissement, de passivité, refuser de travailler ou au contraire faire des efforts pour rester attentif en classe, pour choisir des tâches plus difficiles à réaliser, pour apprendre ses leçons ou encore pour ne pas manquer la classe.

Comme Connell et Wellborn (1991), de nombreux auteurs s'accordent à dire que les trois besoins psychologiques fondamentaux que l'individu cherche à satisfaire sont des déterminants essentiels de sa motivation. C'est le cas notamment des théoriciens de l'autodétermination qui examinent plus en détail, dans leurs travaux, le besoin d'autonomie (par ex.: Deci \& Ryan, 1991; Deci et al., 1991; Ryan \& Connell, 1989; Ryan \& La Guardia, 2000). Ce besoin, selon eux, entretient un lien étroit avec la notion d'internalisation. Cette dernière se définit comme une activité qui consiste à transformer ce que les auteurs nomment "des régulations externes " (valeurs, pratiques, règles sociales et culturelles) en "régulations internes ». Plus simplement, au contact des autres, l'individu s'approprie les valeurs et les croyances qui viennent de son environnement et les fait siennes. Quand I'internalisation 
atteint son plus haut niveau (les théoriciens en définissent quatre), l'individu se perçoit autonome. S'il agit c'est avant tout parce que c'est important pour lui (par ex.: Deci et al., 1991 ; Deci \& Ryan, 1991; Ryan \& Connell, 1989). À l'inverse, lorsque la perception de son autonomie est au niveau le plus bas, la régulation est dite externe. Si la personne mobilise certains efforts c'est avant tout parce qu'elle se sent contrainte de le faire. Pour les théoriciens de l'autodétermination, les différents types de régulations sont prépondérants dans la mesure où ils déterminent le degré de motivation de l'individu. Au plus la personne se perçoit autonome, au plus ses efforts, son implication dans la tâche ou encore sa persistance seront importants. Au plus, en revanche, elle se perçoit contrainte, contrôlée ou surveillée, au moins l'activité proposée présentera pour elle un intérêt. Le continuum d'autonomie va ainsi de pair avec le continuum de motivation.

De nombreuses études menées dans le domaine de l'éducation confirment la pertinence de ces approches en montrant combien les perceptions des élèves peuvent influencer les efforts qu'ils fournissent. Premièrement, concernant le besoin de compétence, les recherches démontrent que lorsque l'élève se perçoit capable de réaliser certaines tâches scolaires non seulement il s'investit davantage dans les activités de classe mais il fournit également des efforts dans ses relations avec les autres. Il déclare aider souvent ses camarades et faire ce que l'enseignant demande (Wentzel, 1996). En revanche, s'il n'attribue pas ses performances à ses propres compétences mais plutôt à des facteurs externes (par ex : « j'ai des bonnes notes simplement parce que mon professeur m'aime bien »), alors cela affecte négativement ses buts sociaux. II fait moins d'efforts pour aider ses camarades, partager avec eux et respecter les règles (Wentzel, 1997).

Deuxièmement, les recherches étudiant, elles, l'autonomie révèlent que lorsque l'élève ne se sent pas contraint, il s'implique davantage dans la tâche proposée (Ryan \& Grolnick, 1986). En favorisant un "origin climate" comme le qualifie De Charms (1976) (c'est-à-dire un climat de classe qui favorise l'avancée de l'individu vers ses propres objectifs) plutôt qu'un « pawn climate " (signifiant « être le pion de quelqu'un"), l'enseignant développe le niveau d'internalisation des élèves. Ces derniers se percevant libres de faire certains choix préfèrent choisir des activités de challenge plutôt que des activités faciles à réaliser et montrent davantage de curiosité face aux tâches proposées (Deci, Nezlek \& Schein- man, 1981). Ryan et Connell (1989) confirment qu'un niveau élevé d'autonomie est lié au but d'apprentissage que l'élève poursuit. En d'autres termes, un élève qui se perçoit autonome cherche avant tout à progresser pour lui-même plutôt que d'obtenir une meilleure note que les autres et recevoir ainsi des jugements favorables.

Enfin, les recherches s'intéressant au besoin de lien de l'élève ont montré que lorsque l'enfant ne se sent pas soutenu par ses camarades, les activités scolaires lui paraissent moins intéressantes (Wentzel \& Asher, 1995). Non seulement sa motivation scolaire est affectée mais également les buts sociaux qu'il poursuit (Wentzel, 1994, 1998 \& 2004). Pourquoi faire des efforts pour coopérer ou partager avec ses camarades alors qu'on pense qu'ils ne nous aiment pas? Concernant sa relation avec l'adulte, les résultats des recherches abondent dans le même sens. Au plus l'enfant pense que l'enseignant lui donne des feedback négatifs, au moins il fait des efforts pour respecter les règles de la classe. À ne pas se sentir soutenu, l'intérêt que l'élève accorde à la discipline enseignée baisse (Midgley, Feldlaufer \& Eccles, 1989). Au plus il pense, au contraire que l'enseignant lui fait confiance et a de hautes attentes le concernant, au plus il est motivé pour agir de manière prosociale (coopérer, partager, aider), et pour respecter les règles de la classe et au plus il est intéressé par l'école (Wentzel, 1997, 1998, 2002b \& 2004). En conséquence et comme le montre cette littérature, lorsque l'élève se pense soutenu par les autres (en termes d'aide, d'encouragements et d'attentes exprimées), les efforts qu'il fournit tant sur le plan scolaire que sur le plan social s'en trouvent positivement influencés.

Dans cette perspective, les deux approches que nous venons de développer ainsi que les nombreuses preuves empiriques qui les étayent nous apportent un éclairage majeur sur les facteurs qui peuvent être à l'origine des buts poursuivis par l'enfant. Elles mettent en exergue que ce ne sont pas les informations réelles que l'environnement transmet qui semblent importantes mais la perception qu'en a l'individu. Autrement dit, seule la réalité qu'il croit percevoir est essentielle dans les efforts qu'il va mobiliser. Ces approches ajoutent que les perceptions de l'individu ne se font pas au hasard mais au regard de trois besoins psychologiques fondamentaux qu'il cherche à satisfaire. En fonction des évaluations faites sur chacun de ces besoins, les efforts mobilisés seront différents. Cependant, une remarque essentielle doit être faite. Les deux 
approches que nous venons de décrire traitent essentiellement de l'impact des perceptions sur la motivation scolaire et non sur la motivation sociale de l'élève. Certaines perceptions (c'est le cas de l'autonomie) n'ont d'ailleurs jamais été étudiées en rapport avec ses buts sociaux. Nos recherches ont donc eu pour objet de répondre à la question suivante: les trois types de perceptions discutées au sein de ces deux approches pourraient-ils expliquer les buts sociaux de l'élève? Plus concrètement, pourrait-on expliquer les efforts que l'enfant fait pour coopérer, partager, aider les autres et respecter les règles par la perception qu'il a de ses compétences sociales, de son autonomie et de ses liens avec les autres?

\section{Une synthèse de recherches}

Pour répondre à cette question jamais abordée auparavant dans la littérature, nous avons mené deux études dont voici une synthèse. Ces deux recherches, de type corrélationnel, ont été conduites auprès de collégiens américains (étude $n^{\circ} 1$ ) et français (étude $\mathrm{n}^{\circ} 2$ ). II était demandé à chacun de ces adolescents de répondre, durant une session de classe, et avec l'autorisation de leurs parents, à tout un ensemble de questions de questions (échelles dite de self-report). Grâce à ces dernières, différents types d'informations ont pu être recueillis.

Dans les deux études, nous avons mesuré les perceptions que les élèves avaient d'eux-mêmes et des autres, dimensions que nous supposions être les déterminants de leurs buts sociaux. Ainsi, la compétence sociale perçue de l'élève était évaluée par des items du type : "Est-ce que comme certains adolescents, tu agis de la façon dont tu es supposé(e) le faire? " ou " Est-ce que comme d'autres, tu n'agis pas comme tu es supposé(e) le faire » (échelle inspirée d'Harter, 1982). Pour évaluer son autonomie perçue, il lui était demandé les raisons qui le poussent à agir. Coopère t-il, par exemple, «Pour éviter de se faire disputer par son professeur?", "Parce que c'est important pour lui? " (items inspirés de l'échelle de Ryan \& Connell, 1989). Enfin, pour évaluer le soutien qu'il pense recevoir de ses pairs et de son enseignant, (ou à quel point il pense que les autres lui font confiance dans ses comportements), il devait répondre à des questions comme: "Mes camarades veulent que je partage mes idées et mon matériel avec eux" et "Mon enseignant veut que j'aide les autres dans leur travail " (échelle adaptée de Johnson et al., 1985).
Le deuxième type d'informations recueillies dans chacune des deux études concernait les buts sociaux poursuivis par les élèves. II leur était alors demandé de répondre à des questions comme: "Est-ce qu'il t'arrive souvent d'aider tes camarades de classe à résoudre un problème que toi tu as compris ? " (exemple d'item prosocial) et "Est-ce qu'il t'arrive souvent de garder le secret des autres ? " (exemple d'item de responsabilité sociale ; Wentzel, 1993)

Soulignons que, d'ores et déjà, grâce à ces mesures, nous pouvions analyser à quel point les perceptions de l'enfant pouvaient prédire ses buts sociaux. Cependant pour être plus complet nous avons ajouté dans les deux études une mesure concernant les comportements de l'enfant avec les autres. En effet, le recueil de ces informations nous permettait de vérifier, si comme dans les travaux antérieurs, les efforts que l'enfant dit fournir (efforts prosociaux et de responsabilité sociale) s'inscrivent de manière réelle dans ses comportements. Nous avons mesuré ces derniers par une technique de nominations des pairs. L'élève avait alors pour tâche de nommer parmi la liste de ses camarades de classe ceux qui coopéraient et partageaient (comportements prosociaux) et ceux qui respectaient les règles (comportements de responsabilité sociale).

Enfin, dans notre seconde recherche, des informations d'ordre académique, non prises en compte dans l'étude 1, ont été recueillies. II nous semblait en effet intéressant d'ajouter une dimension scolaire à nos analyses. En recueillant des informations concernant les performances scolaires de l'enfant et ses buts académiques, nous pouvions notamment examiner si les buts sociaux de l'élève peuvent entretenir un lien direct avec ses performances ou si, au contraire, ces mêmes performances ne s'expliquent que par des efforts fournis dans le domaine académique. Concrètement, à la différence de leurs camarades d'outre-Atlantique, les participants français devaient répondre à des questions supplémentaires comme : "Est-ce qu'il t'arrive souvent d'apprendre de nouvelles choses même si tu n'y es pas obligé(e) ?" (exemple d'item mesurant les buts dits d'apprentissage) et « Est-ce qu'il t'arrive souvent de montrer que tu as appris plus que tes camarades de classe? " (exemple d'item mesurant les buts dits de performance). Les performances scolaires de chaque élève (c'est-à-dire ses notes) nous ont été, elles, communiquées par les professeurs.

Une fois l'ensemble de ces informations recueillies (pour une représentation graphique des liens 
examinés, voire la figure $n^{\circ} 1$ en annexe), des analyses statistiques ont été faites. Plus exactement, dans chacune des deux recherches, nos données ont fait l'objet de deux séries d'analyses de régression. Dans une première série, nous avons examiné à quel point les perceptions de l'enfant déterminaient ses buts. Dans l'étude $n^{\circ} 1$, les variables de perceptions (compétence perçue, autonomie perçue et support social perçu) ont donc été entrées dans l'analyse comme variables prédictrices. Les mesures concernant les buts prosociaux et les buts de responsabilité sociale ont, elles, constitué tour à tour les variables à prédire. Enfin, le lien entre le comportement et les performances a été examiné. Dans l'étude $\mathrm{n}^{\circ} 2$, et avec pour même objectif d'étudier le poids des perceptions sur les buts, les mêmes variables prédictrices ont été également régressées, sur les mesures évaluant les buts académiques de l'enfant (but d'apprentissage et de performance).

Dans la seconde série d'analyse, enfin, nous avons évalué à quel point les buts de l'élève (ses buts sociaux et ses buts académiques) avaient un impact sur ses comportements et sur ses performances scolaires. De fait, les variables de buts (buts sociaux dans l'étude $n^{\circ} 1$ et buts sociaux et académiques dans l'étude $n^{\circ} 2$ ) ont été entrées (en contrôlant leurs déterminants) dans l'analyse de régression en tant que variables prédictrices. Les mesures de comportements (prosociaux et de responsabilité sociale) ainsi que de performances scolaires ont, elles, constitué, tour à tour, les variables à prédire.

Concernant l'impact des perceptions sur les buts, les résultats de ces différentes analyses abondent dans le sens de nos attentes (pour une représentation graphique des résultats, voir la figure $n^{\circ} 2$ en annexe). En effet, de manière générale, ces résultats révèlent que les perceptions de l'enfant prédisent les buts sociaux qu'il poursuit. Plus spécifiquement, dans l'étude $n^{\circ} 1$ comme dans l'étude $n^{\circ} 2$, la perception qu'il a de ses compétences dans le domaine social ainsi que la perception qu'il a des autres (et plus particulièrement de ses camarades) prédisent ses buts prosociaux, c'est-à-dire ses efforts pour aider, coopérer et partager avec les autres. Les efforts qu'il fournit pour respecter les règles (buts de responsabilité sociale) sont prédits, eux, par son autonomie perçue ainsi que la perception de ses compétences. À ces résultats, les analyses menées dans l'étude $\mathrm{n}^{\circ} 2$ sur les buts académiques apportent deux précisions. D'une part, l'autonomie perçue ainsi que le soutien que l'enfant pense recevoir de l'enseignant prédisent également ses efforts pour avoir de meilleures performances que les autres (buts de performance). D'autre part, l'autonomie perçue et ses compétences perçues prédisent les efforts qu'il fournit pour progresser (but d'apprentissages).

Enfin, concernant l'impact des buts sur les performances de l'élève et sur ses comportements, plusieurs résultats peuvent être présentés. Les analyses confirment les études antérieures en révélant que les buts sociaux poursuivis par l'enfant prédisent ses comportements. Autrement dit, les efforts qu'un enfant dit fournir dans ses relations avec les autres s'inscrivent réellement au sein de comportements d'aide, de coopération. Ajoutons que ses comportements prosociaux sont également prédits par les buts de performance. Concernant les résultats scolaires, nos analyses révèlent que les buts sociaux que poursuit l'enfant ne prédisent pas directement ses notes. Ces buts prédisent, nous venons de le voir, les comportements qui eux prédisent les performances en classe. Notons que, réciproquement, les performances scolaires de l'élève jouent un rôle sur l'ensemble de ses comportements. Enfin, une seule dimension motivationnelle prédit les notes obtenues par l'enfant: il s'agit des buts de performance. Autrement dit vouloir être meilleur que les autres, est le seul but qui joue un rôle direct sur les notes que l'enfant obtient à l'école.

Pour résumer, ces résultats révèlent, de manière très intéressante, combien la perception que l'enfant a de lui-même et des autres sont importantes à prendre en considération à l'école. Ces perceptions prédisent les buts qu'il poursuit (tant ses buts sociaux que ses buts académiques) buts qui prédisent ensuite ses apprentissages, de manière directe (c'est le cas des buts de performance) ou de manière indirecte via les comportements qu'il adopte avec les autres (c'est le cas des buts sociaux).

Malgré les apports que ces recherches permettent, quelques limites apparaissent. D'une part, ces recherches sont corrélationnelles. Même si l'on peut estimer le poids que les perceptions peuvent avoir sur les buts, ces études ne nous autorisent pas à établir un lien de cause à effet. Des études expérimentales doivent pour cela être conduites. D'autre part, enfin, bien des dimensions pouvant prédire les buts peuvent encore être étudiées. Dans les recherches ultérieures, on pourra ainsi analyser, comme le suggèrent certains auteurs, à quel point se sentir inclus dans un réseau amical, dans un groupe de référence (Bressoux \& Pansu, 2003 ; Kinderman, 
McCollam \& Gibson, 1996), avec des liens de qualité (Berndt \& Keefe, 1995; Renshaw \& Brown, 1993) peut agir sur les efforts que l'enfant consent à faire dans ses relations avec les autres. Même s'il reste encore à travailler pour améliorer notre connaissance de l'impact et des déterminants des buts sociaux de l'élève à l'école, les résultats de ces deux recherches nous permettent d'ores et déjà d'envisager des implications pour pratique.

\section{IMPLICATIONS POUR LA PRATIQUE}

Pour aider l'enfant à poursuivre des buts sociaux, et l'aider ainsi indirectement dans ses comportements et ses apprentissages, il semble prépondérant de mettre en place des situations pédagogiques qui favoriseraient la satisfaction des trois besoins psychologiques fondamentaux de l'enfant. Dans cette perspective, Connell et Wellborn (1991) suggèrent la mise en place d'une classe dite "structurée". Les informations données en cours doivent être claires. Elles doivent être en quantité suffisante et de bonne qualité. Par les consignes ou les explications qui sont données, l'élève sait exactement ce qui est attendu et les répercussions que tels ou tels comportements peuvent avoir. De ce fait, l'élève peut comprendre ce qu'il faut faire et évaluer avec précision ses compétences. S'il agit dans le sens des attentes, il peut être encouragé et soutenu. S'il les transgresse, il en connaît les risques. Cela ne signifie pas pour autant de la part de l'enseignant qu'il faut se montrer trop rigide quant au respect de ce qu'il faut faire. La transgression doit donner lieu avant tout au dialogue et doit permettre à l'enfant de réfléchir aux conséquences de ses actes. Être trop rigide, ou trop directif quant au comportement des enfants serait aussi le léser dans son besoin d'autonomie (Butera \& Buchs, 2004). II faut suggérer sans imposer, encourager et montrer de intérêt pour ce que fait l'enfant (même en dehors de la classe).

Ces comportements pédagogiques adoptés par déjà la plupart des enseignants peuvent s'accompagner d'une organisation spécifique de la classe " entraînant » l'enfant à certains comportements (l'empathie). Dans cette perspective, différentes méthodes peuvent être envisageables. La première consiste à organiser la classe de manière à faire émerger le conflit socio-cognitif. En amenant ainsi à envisager la perspective de l'autre lors d'activités scolaires, l'enseignant peut aider l'enfant, à en croire la spirale de Doise et Mugny (1997), à apprendre à "se mettre à la place de l'autre » au niveau social. Si l'élève y parvient, il pourra adopter des comportements plus adaptés dans ses relations avec les autres et recevoir des feedback positifs pouvant, en conséquence, améliorer la perception qu'il peut avoir de ses compétences sociales. Par exemple, une méthode nommée " controverse " peut être mise en place (Johnson \& Johnson, 1995). Les élèves sont regroupés en binôme. Chacun est invité à défendre une position sur un sujet donné. L'un sera « pour " et l'autre sera "contre". Non seulement l'un et l'autre devront donner des arguments quant à leur opinion mais ils devront trouver des ripostes qui réfutent les arguments de leur camarade. Les rôles s'inversent, ensuite. La position que l'élève devait défendre devient celle à critiquer en utilisant, bien entendu, une argumentation différente. Enfin, une dernière phase consiste à réconcilier les deux points de vues et à écrire un position qui serait « intégrative " (voir aussi, Buchs, Filisetti et al., 2004). Par cette méthode, l'enfant apprend à se mettre à la place de l'autre et à admettre son point de vue. Deuxièmement, pour favoriser l'adoption de certains comportements en classe, il est possible de mettre en place différentes méthodes d'apprentissage coopératif. Ces méthodes, comme celle dite du "groupe d'experts" (Aronson et al., 1978) mettent l'accent sur ce que les auteurs nomment « l'interdépendance positive" (voir Buchs, Butera, \& Mugny, 2004 ; Buchs, Butera et al., 2004 ; Buchs, Filisetti et al., 2004). II s'agit plus simplement de faire en sorte que les enfants qui doivent travailler ensemble au sein de leur groupe possèdent chacun différentes informations. Pour finaliser le travail demandé, chacun des enfants du groupe a un rôle différent à jouer. Il doit en effet respecter les consignes qui lui sont données, travailler correctement les informations qu'il possède pour mieux les transmettre aux autres, écouter attentivement les informations que ses camarades transmettent pour mieux répondre ensuite aux questions de l'enseignant. Autant d'éléments qui responsabilisent l'élève et qui l'incitent à faire des efforts pour être attentif, coopérer, partager et respecter certaines règles de fonctionnement.

Troisièmement des méthodes d'imitation et de " coaching " peuvent être proposées pour permettre à l'enfant de se faire par exemple des amis pour ensuite avoir des chances d'être soutenu par les autres. En présentant des films démontrant les bons comportements des enfants, les élèves peuvent être incités, selon Hartup (1979) à faire de même. De 
plus, à l'instar des études d'Oden et Asher (1977), il est possible de " coacher " les enfants en les filmant lors d'interactions avec les autres, en leur montrant ensuite leurs comportements, et en discutant avec eux d'une solution pour améliorer leurs agissements.

Par un environnement de classe structuré, favorisant l'autonomie et impliqué, par différentes méthodes pédagogiques l'incitant à adopter certains comportements ou à faire des efforts avec les autres, l'enfant pourraient améliorer certaines perceptions de lui-même et des autres. Celles-ci lui permettraient de s'engager dans la poursuite de buts sociaux notamment, buts jouant un rôle dans ses comportements et par conséquent dans ses apprentissages.

\section{CONCLUSION}

L'école est un lieu où l'opportunité est donnée à l'enfant de poursuivre une multitude de buts. Si la question des buts académiques fait l'objet de nombreuses études, la question des buts sociaux ne doit pas être négligée. En effet, poursuivre des buts sociaux agit sur les apprentissages et les performances en classe, en cela 1) qu'ils sollicitent certaines capacités cognitives qui sont nécessaires aussi à la résolution de problèmes académiques et 2) que poursuivre de tels buts joue un rôle sur l'acceptation par les autres (autres qui fourniront notamment le "coup de pouce" nécessaire pour mieux réussir ou apprendre).

Ainsi, favoriser la poursuite des buts sociaux peut se concevoir comme l'une des pistes à privilégier pour améliorer, de manière indirecte, les apprentis- sages des enfants. Mais comment faire concrètement en classe? Les recherches mettent en évidence que c'est la satisfaction de trois besoins psychologiques fondamentaux (le besoin de compétence, d'autonomie et de lien) qui joue un rôle dans la poursuite de ces buts. Travailler donc à la satisfaction de ces besoins en proposant des situations de classes structurées, où l'implication est forte et où le contrôle est faible sont autant d'éléments pratiques qui sont proposés.

Cependant, beaucoup de travail reste à faire pour déterminer l'ensemble des processus qui peuvent expliquer l'adoption de buts sociaux. Les découvrir permettra d'aider les enfants à se comporter de façon plus adaptée avec les autres, d'être accepté en classe et ainsi de mieux apprendre. Plus généralement, cela permettra de mieux comprendre pourquoi certaines personnes adoptent des comportements que l'on qualifie "d'antisociaux " alors que d'autres adoptent des comportements appropriés. Àl'heure où le "savoir-vivre ensemble" est une priorité de l'école, aider à être bien avec les autres et avec soi-même représente un enjeu social important.

Laurence Filisetti laurence.filisetti@upmf-grenoble.fr Université Pierre Mendès-France (Grenoble 2) Laboratoire de psychologie sociale

Kathryn Wentzel wentzel@umd.edu University of Maryland, College Park (USA)

Éric Dépret eric.depret@upmf-grenoble.fr Université Pierre Mendès France (Grenoble 2) Laboratoire de psychologie sociale

\section{BIBLIOGRAPHIE}

Aronson E. ; Blaney N. ; Stephan C. ; Sikes J. \& Snapp M. (1978). The Jigsaw Classroom. Beverly Hills : Sage.

Austin J. T. \& VANCOUVER J. B. (1996). "Goal constructs in psychology: Structure, process, and content ". Psychological Bulletin, vol. 120 , p. 338-375.

BANDURA A. (1986). Social foundations of thought and action: A social cognitive theory. Englewood Cliffs: Prentice-Hall.

Berndt T. J. \& KeEFE K. (1995). «Friends' influence on adolescents'adjustment to school ». Child Development vol. 66, p. $1312-1329$.
Bressoux P. \& Pansu P. (2003). Quand les enseignants jugent leurs élèves. Paris: PUF.

Buchs C. ; Butera F. \& Mugny G. (2004). « Resource in (ter) dependence, student interactions and performance in cooperative learning ". Educational Psychology, vol. 24 p. 291-314.

Buchs C.; Butera F.; Mugny G. \& Darnon C. (2004). "Conflict resolution and cognitive outcomes. Theory into Practice, vol. 43, p. 23-30.

Buchs C. ; FILISETTI L. ; Butera F. \& QuIAMzADE A. (2004). "Comment l'enseignant peut-il organiser le travail de 
groupe? "In E. Gentaz \& P. Dessus (éd.), Comprendre les apprentissages: sciences cognitives et éducation. Paris: Dunod, p. 167-183.

Butera F. \& Buchs C. (2004). " Autorité et apprentissage: Des objectifs mutuellement exclusifs ? "In M. C. Toczek-Capelle \& D. Martinot (éd.), Le défi éducatif. Paris : A. Colin, p. 227-239.

Colf J. D ; DongF K. A. \& Coppotfi l I, H. (198?). « Dimensions and types of social status: A cross-age perspective $》$ Developmental Psychology, vol. 18, n 4, p. 557-570.

CONNELL J. p. \& WELLBORN J. G. (1991). “ Competence, autonomy, and relatedness: A motivational analysis of selfsystem processes ». In M. R. Gunnar \& L. A. Sroufe (éd.), Self processes and development: The Minnesota Symposia on Child Psychology. Hillsdale: L. Erlbaum, vol. 23, p. 43-78.

DE Charms R. (1976). Enhancing motivation. New York: J. Wiley.

Deci E. L. ; Nezlek J. \& Scheinman L. (1981). « Characteristics of the rewarder and intrinsic motivation of the rewardee ". Journal of Personality and Social Psychology, vol. 40, $n^{\circ} 1$, p. 1-10.

DECI E. L. \& RYAN R. M. (1991). «A motivational approach to self : Integration in personality ». In R. Dienstbier (éd.), Nebraska Symposium on Motivation 1990. Lincoln : University of Nebraska Press, p. 237-288.

Deci E. D. ; Vallerand R. J.; Pelletier L. G. \& Ryan R. M. (1991). "Motivation and Éducation: The self-determination perspective ». Educational Psychologist, vol. 26, $n^{\circ} 3-4$, p. 325-346.

DÉPRET E. \& FILISETTI. L. (2001). « Juger et estimer la valeur d'autrui: Des biais de jugement aux compétences sociales \%. L'Orientation scolaire et professionnelle, vol. 30, n'3, p. 297-315.

Dolse W. \& Mugny G. (1997). Psychologie sociale et développement cognitif. Paris : A. Colin [2 ${ }^{\ominus}$ éd.]

DWECK C. S. (1991). "Self-theories and goals: Their role in motivation, personality, and development ". In R. Dienstbier (éd.), Nebraska symposium on motivation. Lincoln : University of Nebraska Press, vol. 38, p. 199 236.

DWECK C. S. (2002). "The development of ability conceptions ». In A. Wigfield \& J. Eccles (éd.), Development of achievement motivation. New York: Academic Press, p. 57-88.

DWECK C. S. \& LEGGETT E. L. (1988). «A social-cognitive approach to motivation and personality ". Psychological Review, vol. 95, n², p. 256-273.

FINN J. D. \& Rock D. A. (1997). "Academic success among students at risk for school failure ". Journal of Applied Psychology, vol. 82, $\mathrm{n}^{\circ}$ 2, p. 221-234.

FORD M. E. (1982). « Social cognition and social competence in adolescence. Developmental Psychology, vol. 18 , $\mathrm{n}^{\circ} 3$, p. 323-340.

FoRD M. E. (1984). "Linking social-cognitive processes with effective social behavior: A living systems approach ». In P. C. Kendall (éd.), Advances in cognitive-behavioral research and therapy. New York: Academic Press, vol. 3, p. 167-211.

Ford M. E. (1996). "Motivational opportunities and obstacles associated with social responsibility and caring behavior in school contexts ". In J. Juvonen \& K. Wentzel (éd.), Social motivation: Understanding children's school adjustment. New York: Cambridge University Press, p. 126-153.

Gottman J. ; Gonso J. \& Rasmussen B. (1975). « Social interaction, social competence, and friendship in children ». Child Development, vol. 46, p. 709-718.

HARAckiewicz J. M. \& Elliot A. J. (1993). "Achievement goals and intrinsic motivation ". Journal of Personality and Social Psychology, vol. 65, $\mathrm{n}^{\circ}$ 5, p. 904-915.

HARTER S. (1982). «The perceived competence scale for children ". Child Development, vol. 53, p. 87-97.

HARTUP W. W. (1979). "Peer relationship and growth of social competence ». In M. W. Kent \& J. E. Rolf (éd.), Primary prevention of Psychopathology : Social competence in children. New England: University Press of New England, p. 150-170.

HeLton G. B. \& OAKLAND T. D. (1977). «Teacher's attitudinal responses to differing characteristics of elementary school students". Journal of Educational Psychology, vol. $69, n^{\circ} 3$, p. $261-265$.

JOHNSON D. W. \& JOHNSON R. T. (1995). Creative controversy: Intellectual challenge in the classroom. Minneapolis: Interaction Book.

JOHNSON D. W. ; JOHNSON R. T. ; BUCKMAN L. A. \& RICHARDS P S. (1985). "The effect of prolonged implementation of cooperative learning on social support within the classroom ". The Journal of Psychology, vol. 119, $\mathrm{n}^{\circ} 5$, p. 405-411.

Kindermann T. A. ; McCollam T. L. \& Gibson E. (1996). «Peer networks and students'classroom engagement during childhood and adolescence ". In J. Juvonen \& K. Wentzel (éd.), Social motivation: Understanding children's school adjustment. New York: Cambridge University Press, p. 279-312.

Midgley C. ; Feldlaufer H. \& Eccles J. (1989). " Student/ teacher relations and attitudes toward mathematics before and after the transition of junior high school $»$ Child Development, vol. 60, p. 981-992.

Nicholls J. G. (1984). «Achievement motivation: Conception of ability, subjective experience, task choice, and performance ». Psychological Review, vol. 91, p. 328346.

ODEN S. \& ASHER S. R. (1977). " Coaching children in social skills for friendship making". Child Development, vol. 48 , p. $495-506$.

PARKer J. G. \& ASHer S. R. (1987). « Peer relations and later personal adjustment: Are low-accepted children at risk? "Psychological Bulletin, vol. 102, p. 357-389.

PINTRICH p. R. \& Schunk D. H. (1996). Motivation in Education: Theory, research and applications. Englewood Cliffs : Prentice-Hall.

Renshaw P. D. \& Brown P. J. (1993). « Loneliness in middle childhood: Concurrent and longitudinal predictors". Child Development, vol. 64, p. 1271-1284.

RoHRKeMPeR M. M. \& BROPHY J. E. (1983). «Teacher's thinking about problem students". In J. M. Levine \& M. C. Wang (éd.), Teachers and student perceptions: Implications for learning. New-York: L. Erlbaum, p. 29-56. 
Ryan R. M. \& Connell J. P. (1989). «Perceived locus of causality and internalization: Examining the reasons for acting in two domains". Journal of Personality and Social Psychology, vol. 57, n 5, p. 749-761.

Ryan R. M. \& GRolniCK W. S. (1986), " Origins and Pawns in classroom: Self-report and projective assessment of individual differences in children's perceptions". Journal of Personality and Social Psychology, vol. 50, $n^{\circ} 3$, p. 550-558.

RyAN R. M. \& LA GUARDIA J. G. (2000). « What is being optimized ? : Self-determination theory and basic psychological needs". In S. H. Qualls \& N. Abeles (éd.), Psychology and the Aging Revolution. Washington [DC]: American Psychological Association, p. 145-172.

Vosk B. ; Forehand R. ; PARKer J. B. \& Rickard K. (1982). "A multimethod comparison of popular and unpopular children ". Developmental Psychology, vol. 18, $\mathrm{n}^{\circ} 4$, 571-575.

WENTZEL K. R. (1989). « Adolescent classroom goals, standards for performance, and academic achievement : An interactionnist perspective". Journal of Educational Psychology, vol. 81, $\mathrm{n}^{\circ} 2,131-142$.

WENTZEL K. R. (1991a). "Relations between social competence and academic achievement in early adolescence ". Child Development, vol. 62, p. 1066-1078.

WENTZEL K. R. (1991b). "Social and academic goals at school: Motivation and achievement in context". Advances in Motivation and Achievement, vol. 7, p. 185212

WENTZEL K. R. (1993). « Motivation and achievement in early adolescence: The role of multiple classroom goals Journal of Early Adolescence, vol. 13, $n^{\circ} 1$, p. 4-20.

WENTZEL K. R. (1994). «Relations of social goal pursuit to social acceptance, classroom behavior, and perceived social support ". Journal of Educational Psychology, vol. $86, n^{\circ} 2$, p. 173-182.
WENTZEL K. R. (1996). "Social and academic motivation in middle school: Concurrent and long-term relations to academic effort ". Journal of Early Adolescence, vol. 16, $n^{\circ} 4$, p. 390-406.

WENTZEL K. R. (1997). « Student motivation in middle school : The role of perceived pedagogical caring ». Journal of Educational Psychology, vol. 89, $n^{\circ} 3$, p. 411-419.

WENTZEL K. R. (1998). «Social relationship and motivation in middle school: The role of parents, teachers, and peers ". Journal of Educational Psychology, vol. 90, $n^{\circ} 2$, p. 202-209.

WENTZEL K. R. (2002a). "The contribution of social goal setting to children's school adjustment ". In A. Wigfield \& J. Eccles (éd.), The Development of Achievement Motivation. San Diego: US Academic Press, p. 221-246.

WENTZEL K. R. (2002b). "Are effective teachers like good parents ? Interpersonal predictors of school adjustment in early adolescence". Child Development, vol. 73, p. 287-301.

WENTZEL K. R. (2003). "Motivating students to behave in socially competent ways $»$. Theory into Practice, vol. 42 , $n^{\circ} 4$, p. 319-326.

WENTZEL K. R. (2004). « Understanding classroom competence: The role of social-motivational and selfprocesses ". Advances in Child Development, vol. 32, p. 213-241.

WENTZEL K. R. \& Asher S. R. (1995). «The academic lives of neglected, rejected, popular, and controversia children ". Child Development, vol. 66, p. 754-763.

WENTZEL K. R. \& CADWELL K. (1997). "Friendship, peer acceptance, and group membership : Relations to academic achievement in middle school $"$. Child Development, vol. $68, n^{\circ} 6$, p. 1198-1209.

WENTZEL K. R. ; FILISETTI L. \& LoONEY L. (à paraître). Adolescence prosocial behavior: The role of self-processes and contextual cues. [Manuscrit soumis pour publication]. 


\section{ANNEXES}

\begin{tabular}{|c|c|c|}
\hline PEFCEPTIONS & Buts & \\
\hline De soi & Sociaux & COMPONTEMENTS \\
\hline Competerces sociales perçus & Buts prosociaux & Comportements prosocianx \\
\hline Autororrie perçue & Buts de resporbabilité scociale & Comporternents de resporsabilité scuciale \\
\hline Des autres & Acadèmiques & \\
\hline Relation avec les camarades & Buts de performance & PERFORMANCES \\
\hline \multirow[t]{2}{*}{ Relation awe l'erse ignart } & Buts d'appentisage & Perforrainces scolaires \\
\hline & \multicolumn{2}{|c|}{ 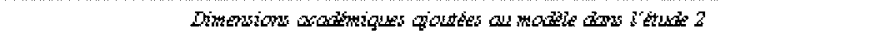 } \\
\hline
\end{tabular}

Figure 1. - Représentation graphique des modèles testés dans nos études

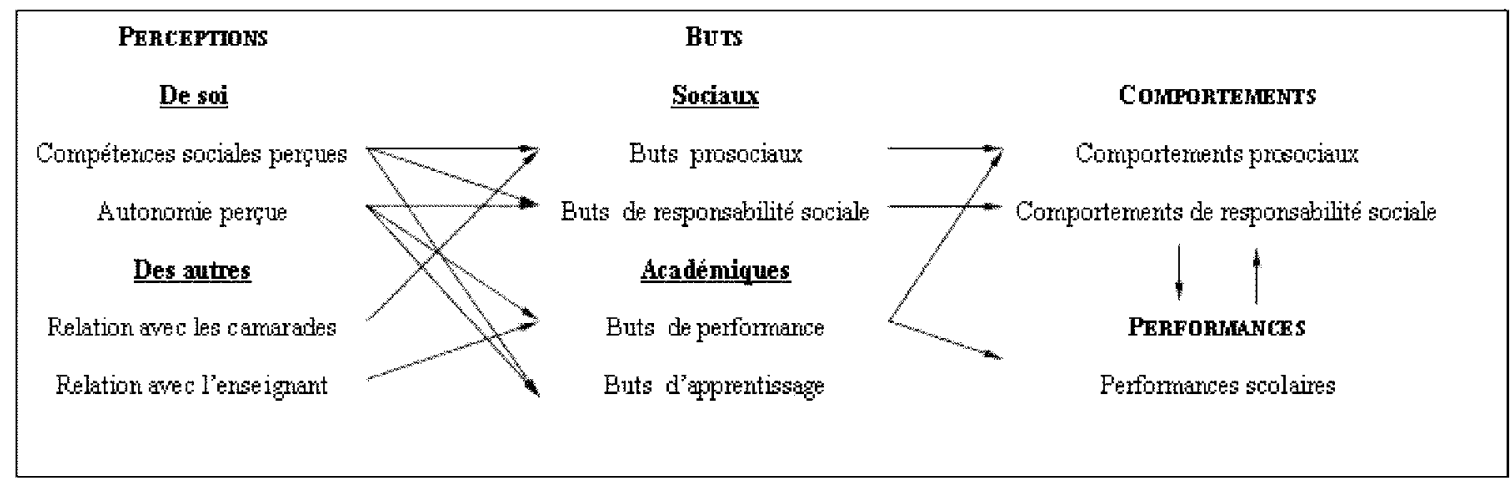

Figure 2. - Représentation graphique des principaux résultats de nos études 